\title{
Patterns of Egg Consumption Can Help Contribute to Nutrient Recommendations and Are Associated with Diet Quality and Shortfall Nutrient Intakes
}

\author{
Yanni Papanikolaou ${ }^{1, *}$ and Victor L. Fulgoni III ${ }^{2}$ (D) \\ 1 Nutritional Strategies, 59 Marriott Place, Paris, ON N3L 0A3, Canada \\ 2 Nutrition Impact, 9725 D Drive North, Battle Creek, MI 49014, USA; vic3rd@aol.com \\ * Correspondence: papanikolaou.yanni@gmail.com; Tel.: +1-519-504-9252
}

Citation: Papanikolaou, Y.; Fulgoni, V.L., III. Patterns of Egg Consumption Can Help Contribute to Nutrient Recommendations and Are Associated with Diet Quality and Shortfall Nutrient Intakes. Nutrients 2021, 13, 4094. https://doi.org/ $10.3390 /$ nu13114094

Academic Editor: Rosa Casas

Received: 8 October 2021

Accepted: 29 October 2021

Published: 16 November 2021

Publisher's Note: MDPI stays neutral with regard to jurisdictional claims in published maps and institutional affiliations.

Copyright: (c) 2021 by the authors. Licensee MDPI, Basel, Switzerland. This article is an open access article distributed under the terms and conditions of the Creative Commons Attribution (CC BY) license (https:/ / creativecommons.org/licenses/by/ $4.0 /)$.

\begin{abstract}
Limited data are available on how eggs are consumed in the typical American eating pattern and the contribution to usual intakes, diet quality and in meeting recommendations. The objectives of the present analysis included identifying how eggs are consumed within U.S. dietary patterns and how these patterns are associated with the usual intakes of shortfall nutrients and diet quality (Healthy Eating Index 2015) using data from the combined National Health and Nutrition Examination Survey (NHANES) from 2001-2016. An additional objective included assessing the differences between egg consumers and egg non-consumers in nutrient intakes and nutrient adequacy. Several egg-containing dietary patterns were identified, and two egg patterns were associated with a greater diet quality compared to a no egg pattern $(p<0.0001)$. Most egg patterns identified were similar in diet quality scores when compared to the no egg pattern; however, the two egg patterns had lower diet quality scores. Egg consumption combined with a greater intake of total protein foods, seafood and plant protein, total vegetables, total fruit, whole fruit, whole grains and dairy foods, and a lower intake of refined grains and added sugars contributed to an improved diet quality, supporting that no one food is responsible for a healthy dietary pattern. Egg consumers demonstrated significantly higher intakes of dietary fiber, calcium, magnesium, potassium, total choline, vitamin A, vitamin C, vitamin D and vitamin $\mathrm{E}$ when compared to egg non-consumers. A comparison of egg consumers and egg non-consumers found egg consumers had significantly less percentages of the population below the EAR for calcium, iron, magnesium, vitamin A, vitamin C and vitamin E. Similarly, the percentage of the population above the recommendations for potassium and choline were greater for egg consumers vs. egg non-consumers. In egg consumers, $24.4 \%$ of the population was above the AI for dietary choline when compared to $4.3 \%$ of egg non-consumers $(p<0.0001)$. Findings from the present analysis demonstrate that eggs and egg-containing foods can be an important part of a healthy dietary pattern when balanced accordingly with other nutrientdense foods.
\end{abstract}

Keywords: NHANES; eggs; usual intakes; shortfall nutrients; dietary patterns

\section{Introduction}

At present, limited data have been published on how Americans consume eggs within dietary patterns. While previous dietary guidance has been critical of the inclusion of eggs within healthy dietary patterns, the current recommendations from both the preceding 2015-2020 Dietary Guidelines for Americans (2015-2020 DGA) [1] and the current 2020-2025 Dietary Guidelines for Americans (2020-2025 DGA) [2] policy reports support egg consumption within dietary patterns and encourage a variety of nutrientdense protein foods, whole grains, dairy products and fruits and vegetables. Furthermore, eggs are nutrient-dense foods when prepared with little or no added solid fats, sugars or sodium, thus further aligning with current dietary guidance [1]. Indeed, the 2020-2025 DGA, for the first time in dietary guidance history, has recommend eggs as a critical 
food for infants and toddlers, in addition to pregnant and lactating women [2], largely due to the choline content provided by eggs [3]. Accumulating evidence has contributed to significant scientific agreement that choline plays an important role in healthy brain development [4-6]. One large egg provides approximately $147 \mathrm{mg}$ of total choline and represents one of the richest sources of dietary choline in the diet [3]. Similarly, the 2020-2025 DGA acknowledges that eggs can fit within the recommended eating patterns for children, adolescents and adults due to their high-quality protein, choline and vitamin contributions, with emphasis on shortfall nutrients, including vitamin D [2].

While dietary guidance offers several recommendations for healthy dietary patterns and promotes the inclusion of high-quality protein foods, at present limited evidence is available that assesses the association of different egg-containing food patterns on diet quality and short fall nutrient intakes. Earlier work examining the associations with egg patterns of consumption and cardiovascular disease risk factors, using data from the National Health and Nutrition Examination Survey (NHANES) 2001-2008, identified eight readily consumed egg patterns (including a no egg pattern) in American adults [7]. Findings revealed that cardiovascular disease risk factors can be influenced by other foods groups consumed with eggs (e.g., fast foods), in addition to the baseline health characteristics (e.g., diabetes) and behavioral choices (e.g., smoking), rather than simply focusing on the inclusion of eggs in the diet [7]. Nonetheless, previous work has not examined the associations between egg dietary patterns of consumption, nutrient intakes and diet quality, and whether eggs can be associated with nutrient adequacy or meeting other nutrient requirements. Thus, the objectives of the present study included identifying how eggs are consumed within U.S. dietary patterns and how these patterns are associated with the usual intakes of shortfall nutrients and diet quality using data from the combined NHANES datasets from 2001 through 2016. An additional objective included assessing the differences between egg consumers and egg non-consumers in nutrient intakes and nutrient adequacy. It is hypothesized that eggs and egg-containing meals can be part of healthy dietary patterns and that consumers of certain egg-containing dietary patterns have better nutrient intakes and diet quality.

\section{Experimental Section}

The National Health and Nutrition Examination Survey (NHANES) is a nationally representative, cross-sectional survey of U.S. noninstitutionalized civilian residents. NHANES data are collected by the National Center for Health Statistics of the Centers for Disease Control and Prevention. Full details of the NHANES have been previously and thoroughly documented in the published literature [8-12]. Written informed consent was obtained for all participants or proxies and the survey protocol was approved by the Research Ethics Review Board at the National Center for Health Statistics. Data from eight NHANES datasets (2001-2002; 2003-2004; 2005-2006; 2007-2008; 2009-2010; 2011-2012; 2013-2014; 2015-2016) were combined for the present analysis in individuals $\geq 2$ years of age. Nutrient intake data for the NHANES are from the relevant United States Department of Agriculture (USDA) Food and Nutrient Database for Dietary Studies (FNDDS) [13]. FNDDS are databases that provide the nutrient values for foods and beverages reported in What We Eat in America (WWEIA) [14], the dietary intake component of the NHANES for each data release. The WWEIA Food Categories provide an application to analyze food and beverages as consumed in the American diet. The classification scheme includes over 150 unique categories, and there are 15 main food groups and 46 subcategories of foods.

WWEIA is collected using the Automated Multiple Pass Method (AMPM). The USDA's AMPM represents a validated dietary data collection instrument that provides an evidence-based, efficient and accurate format for collecting dietary intake data for large-scale national surveys [15]. The AMPM protocol is updated for each 2-year collection of WWEIA to account for the evolving food supply and to address any research needs. The AMPM is a fully computerized recall method that uses a 5-step interview: (1) quick list; (2) forgotten foods; (3) time and occasion; (4) detail cycle; (5) final probe. The AMPM 
includes an extensive compilation of standardized food-specific questions and possible options [15]. Interviewers use dietary recall status codes in both the Individual Foods and Total Nutrient Intakes files to indicate the validity and reliability of responses (i.e., the quality and completeness of a participant's responses) [15].

\subsection{Subjects}

In the present analysis, the combined NHANES dataset sample included male and female participants aged $\geq 2$-years-old who were classified as either egg consumers or egg non-consumers. Subjects with reliable and complete $24 \mathrm{~h}$ dietary recall data from WWEIA were included in the final analysis $(n=65,794)$. Exclusions included pregnant and lactating females and subjects presenting energy intakes equal to zero. Egg consumers were defined as those consuming eggs (i.e., with the exclusion of mixed dishes) during the 24 h dietary recall. Egg intake was determined using food codes in WWEIA category number 2502 'Eggs and omelets', with exclusions for the FNDDS group number 33 'Egg' substitutes' and the FNDDS group number 312 'other poultry eggs'.

Trained individuals completed the $24 \mathrm{~h}$ dietary recalls using the USDA's AMPM, which includes detailed descriptions of all food and amounts consumed by the subjects. As per WWEIA protocols, all participants were eligible for two $24 \mathrm{~h}$ dietary recall interviews. The first dietary recall interview was collected in-person in the Mobile Examination Center (MEC) and the second interview was collected by telephone 3 to 10 days later. Parents or caregivers provided dietary intake information. While 2 days of $24 \mathrm{~h}$ dietary recalls were collected in WWEIA, the current analysis used Day 1 data to define egg consumers and egg non-consumers, as this represents the validated in-person data collection.

\subsection{Methods and Statistical Analysis}

Cluster analyses were used to define various dietary patterns that contain eggs and egg products, while intakes of other food groups (total fruits, total vegetables, whole grains, refined grains and protein food groups) further helped to define the dietary patterns. Egg dietary patterns were identified using SAS 9.4 (SAS Institute, Cary, NC, USA) PROC CLUSTER via a $24 \mathrm{~h}$ dietary recall in the NHANES. Clusters were developed based on the percentage of calories consumed from eggs and egg-containing foods as the centroid for each cluster. Cluster analyses provided the ability to focus on a particular defined aspect (e.g., calories from eggs and egg-containing foods) and then force maximal differences in clusters for assessment. The patterns identified by the cluster analysis were then identified by the percent of calories of each food group (only groups that contributed $5 \%$ or more of calories were used to characterize the clusters).

Total and subcomponents of the Healthy Eating Index 2015 (HEI) scores were used to define diet quality within the dietary patterns. Regression analyses were used to ascertain differences in (1) nutrient adequacy (percentage below the Estimated Average Requirement) and the percentage above the AI of shortfall nutrients as defined previously by dietary guidance [1], including potassium, dietary fiber, choline, magnesium, calcium, iron, and vitamins A, D, E and C, and (2) the total and subcomponent HEI scores. Regression models included age, gender and race/ethnicity as covariates. Usual intakes of shortfall nutrients were determined using the National Cancer Institute's methodology using both days of dietary recall for calculating the usual intakes [16]. All analyses were adjusted for the complex sample design of the NHANES.

\section{Results}

\subsection{Usual Energy and Shortfall Nutrient Intakes in Egg Consumers vs. Egg Non-Consumers}

Egg consumers consumed approximately 109 additional calories per day when compared to egg non-consumers. However, egg consumers demonstrated significantly higher intakes of dietary fiber, calcium, magnesium, potassium, total choline, vitamin A, vitamin C, vitamin $\mathrm{D}$ and vitamin $\mathrm{E}$ when compared to egg non-consumers (see Table 1). 
Table 1. Mean intakes for energy and shortfall nutrients in egg consumers vs. egg non-consumers.

\begin{tabular}{|c|c|c|c|c|c|c|c|c|c|}
\hline Energy/Nutrient & Egg Consumer & Day $1 \mathrm{~N}$ & Day 1 Mean & SE & $p$ & UI N & UI Mean & SE & $p$-Value \\
\hline Energy (kcal) & $\mathrm{NO}$ & 45,791 & 2082 & 7.65 & & 45,798 & 2081 & 6.67 & \\
\hline Energy (kcal) & YES & 20,003 & 2191 & 11.95 & $<0.0001$ & 20,004 & 2190 & 10.65 & $<0.0001$ \\
\hline Dietary fiber (gm) & $\mathrm{NO}$ & 45,791 & 15.6 & 0.11 & & 45,798 & 15.6 & 0.10 & \\
\hline Dietary fiber (gm) & YES & 20,003 & 16.3 & 0.14 & 0.0003 & 20,004 & 16.3 & 0.13 & 0.0001 \\
\hline Calcium (mg) & $\mathrm{NO}$ & 45,791 & 953 & 5.94 & & 45,798 & 954 & 5.18 & \\
\hline Calcium (mg) & YES & 20,003 & 986 & 7.97 & 0.0009 & 20,004 & 986 & 7.18 & 0.0004 \\
\hline Iron (mg) & $\mathrm{NO}$ & 45,791 & 15.0 & 0.08 & & 45,798 & 15.0 & 0.07 & \\
\hline Iron (mg) & YES & 20,003 & 15.2 & 0.11 & 0.1402 & 20,004 & 15.2 & 0.10 & 0.1423 \\
\hline Magnesium (mg) & $\mathrm{NO}$ & 45,791 & 277 & 1.66 & & 45,798 & 277 & 1.50 & \\
\hline Magnesium (mg) & YES & 20,003 & 297 & 2.08 & $<0.0001$ & 20,004 & 297 & 1.86 & $<0.0001$ \\
\hline Potassium (mg) & $\mathrm{NO}$ & 45,791 & 2521 & 13.77 & & 45,798 & 2523 & 11.87 & \\
\hline Potassium (mg) & YES & 20,003 & 2746 & 16.36 & $<0.0001$ & 20,004 & 2747 & 14.84 & $<0.0001$ \\
\hline Total choline (mg) & $\mathrm{NO}$ & 33,500 & 271 & 1.69 & & 33,506 & 270 & 1.36 & \\
\hline Total choline (mg) & YES & 15,673 & 414 & 3.18 & $<0.0001$ & 15,674 & 412 & 2.77 & $<0.0001$ \\
\hline Vitamin A, RAE (mcg) & $\mathrm{NO}$ & 45,791 & 592 & 7.67 & & 45,798 & 591 & 5.87 & \\
\hline Vitamin A, RAE (mcg) & YES & 20,003 & 685 & 7.16 & $<0.0001$ & 20,004 & 686 & 6.37 & $<0.0001$ \\
\hline Vitamin C (mg) & $\mathrm{NO}$ & 45,791 & 81.4 & 1.03 & & 45,798 & 81.1 & 0.92 & \\
\hline Vitamin C (mg) & YES & 20,003 & 90.1 & 1.15 & $<0.0001$ & 20,004 & 89.7 & 1.12 & $<0.0001$ \\
\hline Vitamin D $(\mu \mathrm{g})$ & $\mathrm{NO}$ & 45,791 & 4.62 & 0.04 & & 45,798 & 4.64 & 0.04 & \\
\hline Vitamin D $(\mu \mathrm{g})$ & YES & 20,003 & 5.68 & 0.07 & $<0.0001$ & 20,004 & 5.62 & 0.06 & $<0.0001$ \\
\hline Vitamin $\mathrm{E}$ & $\mathrm{NO}$ & 45,791 & 7.38 & 0.06 & & 45,798 & 7.37 & 0.05 & \\
\hline Vitamin $\mathrm{E}$ & YES & 20,003 & 8.49 & 0.10 & $<0.0001$ & 20,004 & 8.45 & 0.09 & $<0.0001$ \\
\hline
\end{tabular}

Data are from Day 1 recall; vitamin D includes D2 + D3; vitamin E is vitamin E as alpha-tocopherol; U.S. individuals $\geq 2$ years old; gender combined; NHANES 2001-2016. From regression analyses comparing consumer/non-consumer with age, gender and race/ethnicity as covariates.

\subsection{Shortfall Nutrient Adequacy and Inadequacies in Egg Consumers vs. Egg Non-Consumers}

Comparison of egg consumers and egg non-consumers revealed that egg consumers had significantly less percentages of the population below the EAR for calcium, iron, magnesium, vitamin $\mathrm{A}$, vitamin $\mathrm{C}$ and vitamin $\mathrm{E}$ (see Table 2). When considering the percentage of the population above the recommendations for potassium and choline, there was a greater percentage of the population above the AI for egg consumers in comparison to egg non-consumers (see Table 2). For dietary choline, $24.4 \%$ of egg consumers were above the established AI relative to $4.3 \%$ of egg non-consumers $(p<0.0001)$.

Table 2. Percent of U.S. individuals below the EAR and above the AI in egg consumers vs. egg non-consumers.

\begin{tabular}{|c|c|c|c|c|c|c|c|c|c|c|}
\hline Energy/Nutrient & Egg Consumer & $<$ EAR \% & SE & $p$ & $<$ RDA $\%$ & SE & $p$ & $>\mathrm{AI} \%$ & SE & $p$-Value \\
\hline Energy (kcal) & NO & & & & & & & & & \\
\hline Energy (kcal) & YES & & & & & & & & & \\
\hline Dietary fiber (gm) & $\mathrm{NO}$ & & & & & & & 4.94 & 0.25 & \\
\hline Dietary fiber (gm) & YES & & & & & & & 5.49 & 0.41 & 0.2494 \\
\hline Calcium (mg) & $\mathrm{NO}$ & 47.06 & 0.55 & & 66.58 & 0.52 & & & & \\
\hline Calcium (mg) & YES & 43.54 & 0.73 & 0.0001 & 63.47 & 0.72 & 0.0004 & & & \\
\hline Iron (mg) & $\mathrm{NO}$ & 4.92 & 0.19 & & 26.87 & 0.27 & & & & \\
\hline Iron (mg) & YES & 3.07 & 0.13 & $<0.0001$ & 22.85 & 0.30 & $<0.0001$ & & & \\
\hline Magnesium (mg) & $\mathrm{NO}$ & 52.61 & 0.58 & & 71.08 & 0.50 & & & & \\
\hline Magnesium (mg) & YES & 48.28 & 0.80 & $<0.0001$ & 68.71 & 0.68 & 0.0049 & & & \\
\hline Potassium (mg) & NO & & & & & & & 31.17 & 0.60 & \\
\hline Potassium (mg) & YES & & & & & & & 39.17 & 0.77 & $<0.0001$ \\
\hline Total choline (mg) & $\mathrm{NO}$ & & & & & & & 4.31 & 0.19 & \\
\hline Total choline (mg) & YES & & & & & & & 24.41 & 1.17 & $<0.0001$ \\
\hline Vitamin A, RAE (mcg) & $\mathrm{NO}$ & 45.55 & 0.74 & & 70.90 & 0.69 & & & & \\
\hline Vitamin A, RAE (mcg) & YES & 30.72 & 0.92 & $<0.0001$ & 63.23 & 0.83 & $<0.0001$ & & & \\
\hline Vitamin C (mg) & NO & 39.77 & 0.71 & & 50.70 & 0.69 & & & & \\
\hline Vitamin C (mg) & YES & 34.50 & 0.81 & $<0.0001$ & 46.06 & 0.81 & $<0.0001$ & & & \\
\hline Vitamin $\mathrm{D}(\mu \mathrm{g})$ & NO & 94.64 & 0.25 & & 99.42 & 0.06 & & & & \\
\hline Vitamin D $(\mu g)$ & YES & 94.54 & 0.51 & 0.8641 & 99.73 & 0.07 & 0.0005 & & & \\
\hline Vitamin E & $\mathrm{NO}$ & 86.33 & 0.47 & & 94.49 & 0.30 & & & & \\
\hline Vitamin E & YES & 81.06 & 0.83 & $<0.0001$ & 92.32 & 0.56 & 0.0006 & & & \\
\hline
\end{tabular}

Usual intakes determined using two dietary recalls using the National Cancer Institute method (Tooze et al.). SE = standard error; vitamin D includes D2 + D3; vitamin $\mathrm{E}$ is vitamin $\mathrm{E}$ as alpha-tocopherol; $\mathrm{EAR}=$ estimated average requirement; $\mathrm{AI}=$ adequate intake; U.S. individuals $\geq 2$ years old; gender combined; NHANES 2001-2016. From $t$-test comparing consumers/non-consumers. 


\subsection{Dietary Patterns (Clusters of Egg Consumption)}

Table 3 depicts ten dietary patterns of egg consumption and the corresponding sample sizes. Only patterns of consumption with $\geq 200$ subjects were included in the analysis. Cluster number $0(n=52,640)$ represented a no egg consumption dietary pattern. The most popular pattern of consumption (cluster 2 with $10.3 \%$ of calories from eggs, $n=6981$ ) was characterized by a higher percentage of calories from mixed dishes, snacks/sweets and nonalcoholic beverages than in other patterns. The second most popular pattern (cluster 3 with $8.2 \%$ of calories from eggs, $n=2390$ ) was higher in protein foods and vegetables than in other patterns, while the third most popular pattern (cluster 4 with $10.4 \%$ of calories from eggs, $n=1096$ ) was higher in whole grains and fruits than in other patterns.

Table 3. Egg dietary patterns of consumption (clusters) and mean percent calories (kcal) within food groups.

\begin{tabular}{|c|c|c|c|c|c|c|c|c|c|c|c|c|c|c|}
\hline $\begin{array}{l}\text { Cluster } \\
\text { Number }\end{array}$ & $n$ & Eggs & Dairy & $\begin{array}{l}\text { Protein } \\
\text { Foods }\end{array}$ & $\begin{array}{l}\text { Mixed } \\
\text { Dishes }\end{array}$ & $\begin{array}{l}\text { Refined } \\
\text { Grains }\end{array}$ & $\begin{array}{l}\text { Whole } \\
\text { Grains }\end{array}$ & $\begin{array}{l}\text { Snacks/ } \\
\text { Sweets }\end{array}$ & Fruit & Vegetables & $\begin{array}{l}\text { Beverages } \\
\text { Nonalcohol }\end{array}$ & $\begin{array}{l}\text { Beverages } \\
\text { Alcoholic }\end{array}$ & Water & Fats/Oils \\
\hline 0 & 52,460 & 0.00 & 8.14 & 13.42 & 21.57 & 10.45 & 3.30 & 16.31 & 2.81 & 5.23 & 10.27 & 3.11 & 0.07 & 2.99 \\
\hline 1 & 821 & 8.68 & 5.61 & 13.16 & 13.91 & 14.39 & 1.74 & 13.16 & 1.98 & 4.96 & 7.37 & 0.84 & 0.00 & 4.37 \\
\hline 2 & 6981 & 10.33 & 8.45 & 10.66 & 19.59 & 11.86 & 1.80 & 15.92 & 2.46 & 3.85 & 10.72 & 0.77 & 0.00 & 2.38 \\
\hline 3 & 2390 & 8.21 & 4.61 & 26.84 & 7.55 & 9.16 & 1.31 & 11.75 & 2.11 & 13.60 & 7.54 & 2.93 & 0.00 & 3.10 \\
\hline 4 & 1096 & 10.38 & 9.35 & 12.56 & 9.36 & 9.71 & 12.70 & 9.85 & 8.16 & 5.63 & 6.68 & 1.14 & 0.01 & 2.63 \\
\hline 5 & 538 & 7.36 & 5.93 & 11.80 & 14.61 & 10.71 & 2.38 & 12.98 & 2.01 & 5.27 & 5.82 & 2.42 & 0.01 & 17.31 \\
\hline 6 & 790 & 8.03 & 4.26 & 11.99 & 17.26 & 7.85 & 1.54 & 8.29 & 0.96 & 3.83 & 6.69 & 25.31 & 0.01 & 2.60 \\
\hline 7 & 518 & 8.84 & 5.68 & 13.30 & 12.41 & 12.30 & 2.00 & 13.53 & 2.06 & 5.11 & 7.60 & 4.80 & 0.01 & 2.89 \\
\hline
\end{tabular}

Data from Day 1 recall from NHANES 2001-2016. Proc Cluster of SAS used to define clusters (only those with sample size $>200$ retained).

WWEAI food groups were used to develop clusters based on \% calories from each food group with eggs as the centroid of the cluster.

\subsection{Egg Dietary Patterns of Consumption and Diet Quality}

Table 4 depicts HEI total and subcomponent scores within each egg cluster group. Several egg-containing dietary patterns had total HEI scores that were not significantly different when compared to the no egg dietary pattern. Total HEI scores for clusters 1 and 2 were significantly lower than cluster 0 (no eggs). The cluster 1 dietary pattern had lower intakes from total vegetables, greens and beans, whole grains and dairy, and higher intakes of saturated fat and added sugars when compared to the no eggs pattern. Cluster 2 represented lower intakes of total vegetables, whole grains, seafood and plant protein and added sugars, and greater intakes of saturated fat relative to the no eggs pattern of consumption. In contrast, clusters 3 and 4 had total HEL scores that were significantly greater when compared to cluster 0 (no eggs). Subcomponent HEI scores in cluster 3 were significantly greater for total protein foods, seafood and plant protein, total vegetables, total fruit, whole fruit, whole grains and dairy, and lower intakes of added sugars and refined grains, but also lower for sodium. Individuals in the cluster 4 pattern had $10.4 \%$ of total calories sourced from eggs. Cluster 4 individuals also had greater scores from higher consumption of total fruit, whole fruit, whole grains, greens and beans, dairy foods and total protein foods, and higher scores from lower intakes of refined grains, saturated fat and added sugars. This implies that eggs and egg-containing foods can be an important part of a healthy dietary pattern when balanced accordingly with other nutrient-dense food categories. 
Table 4. Least square mean total and subcomponent Healthy Eating Index (2015) scores by egg cluster in Americans.

\begin{tabular}{|c|c|c|c|c|c|c|c|c|c|c|c|c|c|c|c|c|c|c|c|c|c|c|c|c|}
\hline \multirow{2}{*}{ Variable } & \multicolumn{3}{|c|}{ Cluster 0} & \multicolumn{3}{|c|}{ Cluster 1} & \multicolumn{3}{|c|}{ Cluster 2} & \multicolumn{3}{|c|}{ Cluster 3} & \multicolumn{3}{|c|}{ Cluster 4} & \multicolumn{3}{|c|}{ Cluster 5} & \multicolumn{3}{|c|}{ Cluster 6} & \multicolumn{3}{|c|}{ Cluster 7} \\
\hline & LSM & SE & $p$ & LSM & SE & $p$ & LSM & SE & $p$ & LSM & SE & $p$ & LSM & SE & $p$ & LSM & SE & $p$ & LSM & SE & $p$ & LSM & SE & $p$ \\
\hline HEI-2015 component 1 (total vegetables) & 2.85 & 0.01 & $<0.0001$ & 2.33 & 0.08 & $<0.0001$ & 2.69 & 0.04 & 0.0001 & 3.48 & 0.05 & $<0.0001$ & 2.92 & 0.09 & 0.4079 & 3.39 & 0.10 & $<0.0001$ & 2.42 & 0.08 & $<0.0001$ & 3.43 & 0.10 & $<0.0001$ \\
\hline HEI-2015 component 2 (greens and beans) & 1.29 & 0.02 & $<0.0001$ & 1.00 & 0.10 & $<0.0001$ & 1.34 & 0.05 & 0.2776 & 1.45 & 0.08 & 0.0572 & 1.57 & 0.12 & 0.0302 & 1.64 & 0.16 & 0.0309 & 1.26 & 0.09 & 0.7433 & 1.86 & 0.16 & 0.0003 \\
\hline HEI-2015 component 3 (total fruit) & 2.15 & 0.02 & $<0.0001$ & 2.00 & 0.11 & 0.1599 & 2.32 & 0.05 & 0.0001 & 2.00 & 0.06 & 0.0130 & 3.37 & 0.11 & $<0.0001$ & 1.95 & 0.13 & 0.1360 & 1.44 & 0.10 & $<0.0001$ & 1.99 & 0.14 & 0.2495 \\
\hline HEI-2015 component 5 (whole grains) & 2.36 & 0.03 & $<0.0001$ & 1.68 & 0.14 & $<0.0001$ & 1.84 & 0.06 & $<0.0001$ & 1.44 & 0.08 & $<0.0001$ & 6.61 & 0.16 & $<0.0001$ & 1.97 & 0.17 & 0.0209 & 1.44 & 0.13 & $<0.0001$ & 2.01 & 0.19 & 0.0682 \\
\hline HEI-2015 component 6 (dairy) & 5.60 & 0.03 & $<0.0001$ & 4.90 & 0.16 & $<0.0001$ & 5.94 & 0.07 & $<0.0001$ & 4.07 & 0.10 & $<0.0001$ & 6.29 & 0.14 & $<0.0001$ & 4.99 & 0.19 & 0.0030 & 4.27 & 0.14 & $<0.0001$ & 5.33 & 0.22 & 0.2240 \\
\hline HEI-2015 component 7 (total protein foods) & 3.92 & 0.01 & $<0.0001$ & 4.37 & 0.05 & $<0.0001$ & 4.46 & 0.02 & $<0.0001$ & 4.70 & 0.02 & $<0.0001$ & 4.42 & 0.05 & $<0.0001$ & 4.36 & 0.05 & $<0.0001$ & 4.34 & 0.05 & $<0.0001$ & 4.46 & 0.06 & $<0.0001$ \\
\hline $\begin{array}{l}\text { HEI-2015 component } 8 \text { (seafood and plant } \\
\text { protein) }\end{array}$ & 2.09 & 0.02 & $<0.0001$ & 1.86 & 0.13 & 0.0723 & 1.98 & 0.05 & 0.0199 & 2.37 & 0.08 & 0.0013 & 2.20 & 0.11 & 0.3152 & 1.70 & 0.13 & 0.0021 & 1.94 & 0.11 & 0.2045 & 2.37 & 0.16 & 0.0784 \\
\hline HEI-2015 component 9 (fatty acid ratio) & 4.66 & 0.03 & $<0.0001$ & 4.51 & 0.20 & 0.4435 & 4.19 & 0.07 & $<0.0001$ & 5.86 & 0.12 & $<0.0001$ & 5.09 & 0.17 & 0.0123 & 5.78 & 0.21 & $<0.0001$ & 4.64 & 0.15 & 0.8882 & 4.97 & 0.24 & 0.1836 \\
\hline HEI-2015 compor & 5.80 & 0.03 & $<0.0001$ & 6.04 & 0.20 & 0.2249 & 5.87 & 0.08 & 0.4011 & 7.90 & 0.07 & $<0.0001$ & 7.96 & 0.13 & $<0.0001$ & 6.65 & 0.20 & 0.0001 & 7.59 & 0.13 & $<0.0001$ & 6.34 & 0.23 & 0.0216 \\
\hline HEI-2015 component 12 (saturated fat) & 6.09 & 0.03 & $<0.0001$ & 5.16 & 0.18 & $<0.0001$ & 4.72 & 0.07 & $<0.0001$ & 4.89 & 0.11 & $<0.0001$ & 6.63 & 0.15 & 0.0004 & 3.50 & 0.18 & $<0.0001$ & 6.84 & 0.15 & $<0.0001$ & 4.87 & 0.23 & $<0.0001$ \\
\hline HEI-2015 component 13 (added & 6.07 & 0.04 & $<0.0001$ & 4.78 & 0.16 & $<0.0001$ & 6.48 & 0.09 & $<0.0001$ & 7.57 & 0.10 & $<0.0001$ & 7.61 & 0.13 & $<0.0001$ & 7.62 & 0.17 & $<0.0001$ & 7.80 & 0.11 & $<0.0001$ & 7.30 & 0.19 & $<0.0001$ \\
\hline HEI-2015 Total Score & 49.51 & 0.16 & $<0.0001$ & 44.89 & 0.63 & $<0.0001$ & 47.63 & 0.29 & $<0.0001$ & 50.93 & 0.40 & 0.0007 & 62.24 & 0.62 & $<0.0001$ & 48.22 & 0.83 & 0.1269 & 50.49 & 0.52 & 0.0645 & 49.94 & 0.92 & 0.6329 \\
\hline
\end{tabular}

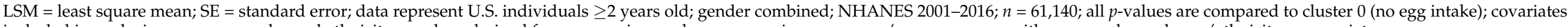
included in analysis were age, gender and ethnicity; $p$-values derived from regression analyses comparing consumer/non-consumer with age, gender and race/ethnicity as covariates. 


\section{Discussion}

To our knowledge, this is the first study using the NHANES data to identify egg patterns of consumption in the American population. Our findings identified several egg cluster patterns within the population, with two egg patterns of consumption associated with improved diet quality scores and lower diet quality scores relative to the no egg consumption pattern. All the other egg patterns of consumption that were identified had diet quality scores that were similar to the no egg pattern. The two egg patterns that showed the greatest diet quality scores also had greater intakes of nutrient-dense foods, including greater intakes of total protein foods, seafood and plant protein, total vegetables, total fruit, whole fruit and whole grain, and lower intakes of refined grains and added sugars. These preliminary data imply that eggs can be part of a healthy dietary pattern, particularly an eating pattern that is inclusive of nutrient-rich foods. Furthermore, the current data also support that diet quality changes are not due to eggs, but rather the foods that are consumed with eggs and within the whole of the dietary pattern. The current study also assessed intakes of shortfall nutrients with findings showing that egg consumers have significantly higher intakes of dietary fiber, calcium, magnesium, potassium, total choline, vitamin A, vitamin C, vitamin D and vitamin E when compared to egg nonconsumers. Similarly, the data supports that a smaller percentage of egg consumers are below recommendations for calcium, iron, magnesium, vitamin A, vitamin $\mathrm{C}$ and vitamin $\mathrm{E}$ when compared to egg non-consumers, though some of the changes, while statistically significant, were small. Furthermore, a higher percentage of the population meet the AI for total choline when comparing egg consumers to egg non-consumers.

The present findings align with our previous work and that of other researchers studying eggs, nutrients and health-related outcomes. An NHANES study examining egg consumption and the links to nutrient intakes and growth-related outcomes in an infant population showed egg consumption was associated with higher nutrient intakes when compared to infant egg non-consumers. Specifically, egg consumption was associated with significantly higher intakes of protein, lutein + zeaxanthin, choline, B12, selenium and phosphorus, and lower intakes of added and total sugars per day relative to egg nonconsumers [17]. Similarly, our previous NHANES analyses in children and adolescents found that an eating pattern that included eggs was linked with higher amounts of several nutrients, including protein, polyunsaturated and monounsaturated fat, $\alpha$-linolenic acid, DHA, lutein + zeaxanthin, potassium, phosphorus, choline, riboflavin, selenium, choline and vitamins D, E and A. Egg consumers also consumed less total and added sugars relative to children and adolescents consuming a no egg dietary pattern [18].

Previous literature has recognized the dietary significance of choline largely due to its relevance in metabolism and physiology [4,5], with several publications targeting the critical role choline plays in neuronal structures in early life [19-22]. Choline can be generated endogenously, but the amounts produced do not support physiological requirements $[5,23]$. Eggs have been identified as a leading dietary source of choline, with a $50 \mathrm{~g}$ hard-boiled egg contributing $146.9 \mathrm{mg}$ total choline or $27 \%$ of the recommended daily value [3]. The majority of American children consume less than the AI (550 mg for individuals greater than 4 years of age) [5]. It has previously been reported that the average choline intake in children and adolescents was approximately $256 \mathrm{mg}$ per day [5]. A recent modeling analysis by our group has corroborated previous findings that have demonstrated younger populations are not meeting the established recommendations for choline. Concurrently, the same analysis showed that eggs can help to reduce the shortfall gaps in choline intake and improve the likelihood of meeting the recommendations in younger Americans. Overall, modeling the removal of eggs from the diet in younger Americans decreased the intakes of choline, thereby resulting in fewer participants above the AI for choline. In contrast, the addition of eggs to the weekly eating pattern resulted in substantial increases in choline intakes and more individuals above the AI for choline [18]. Likewise, choline plays an important role in adult health, representing an integral role in the 
structural integrity of cell membranes, methyl metabolism, cholinergic neurotransmission, transmembrane signaling, lipid and cholesterol transport and metabolism [23]. Among U.S. adults $\geq 19$ years old, as well as pregnant women, less than $10 \%$ meet the AI for choline [5]. Indeed, U.S. adults consuming eggs were more likely to meet the recommendations for AI relative to egg non-consumers. Furthermore, egg consumers had approximately double the usual intake of choline relative to egg non-consumers. Adults including eggs in their dietary pattern also had slightly elevated diet quality scores [7], thus aligning with the findings of the current analysis.

The current analyses have limitations inherent in observational research and have been reported in numerous previously published, peer-reviewed studies [24-26]. The results are dependent on self-reported dietary data for foods, which may involve study participants under- or over-estimating food consumption, thereby leading to inaccuracies in energy and nutrient intakes. Data were also obtained using a $24 \mathrm{~h}$ dietary recall, which relies on the study caregiver's memory, and while the validated methods are used to gather the data, the recall information is subject to inaccuracies and bias from memory challenges and other potential measurement errors experienced in epidemiological investigations [27,28]. Our current analysis considered the dietary patterns with and without egg consumption, so other food choices within an individual's eating pattern may also contribute to the relationships observed with nutrient intakes. A significant benefit of using the NHANES data for the current analyses include access to a large and nationally representative dataset of adults of various age groups in the U.S. with the corresponding food and nutrient intake data. As the present research is observational, and since growth and development are multifactorial, future research designs will need to consider randomized controlled trials.

\section{Conclusions}

Our analysis has identified that several egg patterns of consumption are routine in the American population. The egg patterns demonstrating the higher diet quality scores compared to a no egg food pattern included greater consumption of nutrient-dense foods, including greens and beans, total fruit, whole fruit, whole grains, dairy, total protein foods and seafood and plant protein. The current data support that eggs can be part of a healthy dietary pattern, particularly an eating pattern that is inclusive of nutrient-dense foods, thereby supporting the notion that foods should not be considered in isolation but rather as part of an entire dietary pattern. Nutrient intake analyses further showed that egg consumers have significantly higher intakes of dietary fiber, calcium, magnesium, potassium, total choline, vitamin A, vitamin C, vitamin D and vitamin E when compared to egg non-consumers. The current data assessing usual intakes also suggest that a smaller percentage of egg consumers are below the recommendations for calcium, iron, magnesium, vitamin A, vitamin C and vitamin E when compared to egg non-consumers. Additionally, when including eggs in the diet, a greater percentage of the population meet the AI for total choline relative to egg non-consumers. The present findings are aligned with the previously published data documenting several benefits associated with egg consumption.

Author Contributions: Y.P. and V.L.F.III collaborated on the intellectual conception and interpretation of the research; V.L.F.III developed the design of the research and conducted the final analyses; Y.P. drafted the manuscript. All authors have read and agreed to the published version of the manuscript.

Funding: This research was funded by the Egg Nutrition Center in January 2021.

Institutional Review Board Statement: The studies involving human participants were reviewed and approved by the Research Ethics Review Board at the National Center for Health Statistics.

Informed Consent Statement: Written informed consent to participate in this study was provided by the participants' legal guardian/next of kin as per guidelines established by the Research Ethics Review Board at the National Center for Health Statistics.

Data Availability Statement: Publicly available datasets were analyzed in this study. This data can be found here: https: / / wwwn.cdc.gov/nchs/nhanes (accessed on 16 July 2021). 
Acknowledgments: The Egg Nutrition Center provided a research honorarium to help fund and support the present analysis of NHANES.

Conflicts of Interest: The funding sponsors had no role in the design of the study; in the collection, analyses or interpretation of data; in the writing of the manuscript or in the decision to publish the results. Y.P., as Vice President of Nutritional Strategies, provides food, nutrition and regulatory affairs consulting services for food and beverage companies and food-related associations, and collaborates with V.L.F.III on the NHANES analyses. V.L.F.III, as Senior Vice President of Nutrition Impact, provides food and nutrition consulting services for food and beverage companies. V.L.F.III also conducts analyses of NHANES data for members of the food industry.

\section{References}

1. U.S. Department of Health and Human Services; U.S. Department of Agriculture. 2015-2020 Dietary Guidelines for Americans, 8th ed.; 2015. Available online: https:/ / health.gov/our-work/food-nutrition/previous-dietary-guidelines/2015 (accessed on 1 October 2021).

2. U.S. Department of Agriculture; U.S. Department of Health and Human Services. Dietary Guidelines for Americans, 2020-2025, 9th ed.; 2020. Available online: DietaryGuidelines.gov (accessed on 1 October 2021).

3. United States Department of Agriculture. Agricultural Research Service USDA Food Composition Databases, USDA National Nutrient Database for Standard Reference (Release 29, Released September 2015, Revised April 2018). Food Group: Dairy and Egg Products: Reference No. 01123, Egg, Whole, Raw, Fresh. Available online: https://ndb.nal.usda.gov/ndb/ (accessed on 23 September 2021).

4. Zeisel, S.H.; da Costa, K.-A. Choline: An essential nutrient for public health. Nutr. Rev. 2009, 67, 615-623. [CrossRef] [PubMed]

5. National Institutes of Health; Office of Dietary Supplements; Choline. Choline-Health Professional Fact Sheet. Available online: Nih.gov (accessed on 10 January 2021).

6. Weideman, A.M.; Barr, S.I.; Green, T.J.; Xu, Z.; Innis, S.M.; Kitts, D.D. Dietary choline intake: Current state of knowledge across the life cycle. Nutrients 2018, 10, 1513. [CrossRef] [PubMed]

7. Nicklas, T.A.; O’Neil, C.E.; Fulgoni, V.L., III. Differing statistical approaches affect the relation between egg consumption, adiposity, and cardiovascular risk factors in adults. J. Nutr. 2015, 145, 170S-176S. [CrossRef] [PubMed]

8. US Centers for Disease Control and Prevention; National Center for Health Statistics. National Health and Nutrition Examination Survey. NHANES-National Health and Nutrition Examination Survey Homepage. Available online: cdc.gov (accessed on 27 July 2021).

9. Centers for Disease Control and Prevention; National Center for Health Statistics. National Health and Nutrition Examination Survey. Analytic and Reporting Guidelines. Available online: https://wwwn.cdc.gov/nchs/nhanes/analyticguidelines.aspx (accessed on 27 July 2021).

10. Centers for Disease Control and Prevention; National Center for Health Statistics. National Health and Nutrition Examination Survey. NHANES 2011-2012. Available online: https:/ /wwwn.cdc.gov/nchs/nhanes/ContinuousNhanes/Overview.aspx? Begin Year=2011 (accessed on 26 July 2021).

11. Centers for Disease Control and Prevention; National Center for Health Statistics. National Health and Nutrition Examination Survey. NHANES 2013-2014. Available online: https:/ /wwwn.cdc.gov/nchs/nhanes/ContinuousNhanes/Overview.aspx? Begin Year=2013 (accessed on 18 July 2021).

12. Papanikolaou, Y.; Miller-Jones, J.; Fulgoni, V.L. Several grain dietary patterns are associated with better diet quality and improved shortfall nutrient intakes in US children and adolescents: A study focusing on the 2015-2020 Dietary Guidelines for Americans. Nutr. J. 2017, 16, 1-10. [CrossRef] [PubMed]

13. US Department of Agriculture; Agricultural Research Service; Food Surveys Research Group. Food and Nutrient Database for Dietary Studies. Available online: https:/ / www.ars.usda.gov/northeast-area/beltsville-md-bhnrc/beltsville-human-nutritionresearch-center/food-surveys-research-group/docs/fndds-download-databases/ (accessed on 16 July 2021).

14. United States Department of Agriculture; Agricultural Research Service; Food Service Research Group. What We Eat in America, National Health and Nutrition Examination Survey Overview, 2001-2018: Data Collection; United States Department of Agriculture, Agricultural Research Service: Beltsville, MD, USA, 2021. Available online: https:/ / www.ars.usda.gov/northeast-area/beltsvillemd-bhnrc/beltsville-human-nutrition-research-center/food-surveys-research-group/docs/wweianhanes-overview/ (accessed on 29 August 2021).

15. Moshfegh, A.J.; Rhodes, D.G.; Baer, D.J.; Murayi, T.; Clemens, J.C.; Rumpler, W.V.; Paul, D.R.; Sebastian, R.S.; Kuczynski, K.C.; Ingwersen, L.A.; et al. The USDA Automated Multiple-Pass Method reduces bias in the collection of energy intakes. Am. J. Clin. Nutr. 2008, 88, 324-332. [CrossRef]

16. National Cancer Institute; Division of Cancer Control and Population Sciences. Usual Dietary Intakes: The NCI Method. Available online: https:/ / epi.grants.cancer.gov / diet/usualintakes/method.html (accessed on 11 June 2021).

17. Papanikolaou, Y.; Fulgoni, V.L. Egg consumption in U.S. children is associated with greater daily nutrient intakes, including protein, lutein + zeaxanthin, choline, linolenic acid, and docosahexanoic acid. Nutrients 2019, 11, 1137. [CrossRef] 
18. Papanikolaou, Y.; Fulgoni, V.L. Modeling the removal and addition of eggs in the current US Diet is linked to choline and lutein + zeaxanthin usual intakes in childhood. Curr. Dev. Nutr. 2021, 5, nzaa181. [CrossRef]

19. Mun, J.G.; Legette, L.L.; Ikonte, C.J.; Mitmesser, S.H. Choline and DHA in maternal and infant nutrition: Synergistic implications in brain and eye health. Nutrients 2019, 11, 1125. [CrossRef] [PubMed]

20. Ueland, P.M. Choline and betaine in health and disease. J. Inherit. Metab. Dis. 2010, 34, 3-15. [CrossRef] [PubMed]

21. Bekdash, R.A. Neuroprotective effects of choline and other methyl donors. Nutrients. 2019, 11, 2995. [CrossRef] [PubMed]

22. Bekdash, R.A. Choline and the brain: An epigenetic perspective. Adv. Neurobiol. 2016, 12, 381-399. [PubMed]

23. Zeisel, S.H. Choline. In Modern Nutrition in Health and Disease, 11th ed.; Ross, A.C., Caballero, B., Cousins, R.J., Tucker, K.L., Ziegler, T.R., Eds.; Lippincott Williams \& Wilkins: Baltimore, MD, USA, 2014; pp. 416-426.

24. Papanikolaou, Y.; Fulgoni, V.L. Egg Consumption in Infants is Associated with Longer Recumbent Length and Greater Intake of Several Nutrients Essential in Growth and Development. Nutrients 2018, 10, 719. [CrossRef] [PubMed]

25. Ahluwalia, N.; Dwyer, J.; Terry, A.; Moshfegh, A.; Johnson, C. Update on NHANES Dietary Data: Focus on Collection, Release, Analytical Considerations, and Uses to Inform Public Policy. Adv. Nutr. Int. J. 2016, 7, 121-134. [CrossRef] [PubMed]

26. Zipf, G.; Chiappa, M.; Porter, K.; Ostchega, Y.; Lewis, B.; Dostal, J. The National Health and Nutrition Examination Survey: Plans and operations. Vital Health Stat. 2013, 56, 1-37.

27. Grandjean, A.C. Dietary intake data collection: Challenges and limitations. Nutr. Rev. 2012, 70, S101-S104. [CrossRef] [PubMed]

28. Ferrari, P.; Slimani, N.; Ciampi, A.; Trichopoulou, A.; Naska, A.; Lauria, C.; Veglia, F.; Bueno-de-Mesquita, H.B.; Ocké, M.C.; Brustad, M. Evaluation of under- and overreporting of energy intake in the 24-hour diet recalls in the European Prospective Investigation into Cancer and Nutrition (EPIC). Public Health Nutr. 2002, 5, 1329-1345. [CrossRef] [PubMed] 